\title{
Sociologie clinique et santé mentale
}

\section{Vincent de Gaulejac* \\ \& Diane Laroche**}

Résumé: Le projet "Rétablissement et projet de vie" a un double objectif: $i$. proposer aux intervenants et aux résidents de la Maison Saint-Dominique (MSD - Montréal/Canada), un travail approfondi sur leur "roman familial et trajectoire sociale", dans l'orientation de la sociologie clinique; ii. développer une recherche-action pour évaluer cette expérimentation en tant qu'approche alternative en santé mentale, la diffuser et la confronter à d'autres pratiques dans le domaine de la santé mentale. Le texte qui suit présente, dans un premier temps, la démarche, ses soubassements théoriques et méthodologiques. Dans un deuxième temps, il rend compte de la façon dont les participants l'ont mis en œuvre et les effets perceptibles à partir de leurs témoignages. La démarche illustre l'intérêt de développer une clinique de la complexité dans le domaine de la santé mentale.

Mots clés: Sociologie clinique. Maison Saint-Dominique. Santé mentale. Clinique de la complexité. Soutien psychosocial.

\section{Clinical sociology and mental health}

Abstract: The project "Recovery and life project" has a double objective: i. propose to the employees and residents of Maison Saint-Dominique (MSD - Montréal/Canadá), an in-depth work on his "family history and social trajectory", under the guidance of clinical sociology, ii. develop an action research to evaluate this experiment as an alternative perspective on mental health, to be disseminated and compared other practices in the field of mental health. The article presents, in the first moment, the approach, its theoretical and methodological foundations. In the second moment, it reports the way in which the participants carried out the proposal and the perceived effects from their testimonies. The approach illustrates the interest in developing a clinic of complexity within the domain of mental health.

Keywords: Clinical sociology. Maison Saint-Dominique. Mental health. Complexity clinic. Psychosocial support

\section{Sociologia clínica e saúde mental}

Resumo: O projeto "Recuperação e projeto de vida" apresenta um duplo objetivo: i. propor aos funcionários e aos residentes da Maison Saint-Dominique (MSD - Montréal/Canadá), um trabalho aprofundado sobre sua "história familiar e trajetória social", sob a orientação da sociologia clínica; ii. desenvolver uma pesquisa-ação para avaliar esse experimento como perspectiva alternativa em saúde mental, a difundir e a confrontar com outras práticas no domínio da saúde mental. O texto

\author{
* Vincent de \\ Gaulejac est \\ sociologue, \\ professeur émérite \\ de sociologie à l' \\ Unité de Formation \\ et de Recherche \\ (UFR) de Sciences \\ Sociales de \\ I'Université Paris \\ -Diderot. II est \\ l'auteur d'une \\ vingtaine d'ouvrages \\ et anime la collection \\ sociologie clinique \\ chez les Éditions \\ Érès. II a dirigé \\ le Laboratoire \\ de Changement \\ Social de 1981 à \\ 2014. Membre \\ fondateur du Réseau \\ International de \\ Sociologie Clinique, \\ il est l'un des \\ principaux initiateurs \\ de cette orientation \\ scientifique qui \\ s'intéresse aux \\ relations entre \\ l'être de l'homme, \\ l'être de la société \\ et à la dimension \\ existentielle des \\ rapports sociaux. \\ Il a développé des \\ groupes d'implication \\ et de recherche en \\ Europe, Amérique du \\ Nord et du Sud. \\ Orcid: 0000-0002- \\ 9273-5877. \\ $<$ v.gaulejac@ \\ wanadoo.fr> \\ ** Diane Laroche est \\ vice-présidente du \\ Réseau Québécois \\ pour la Pratique \\ des Histoires de Vie \\ (RQPHV). Sociologue \\ clinicienne et
}


membre du Réseau International de Sociologie Clinique (Risc). Praticienne et formatrice avec l'approche roman familial et trajectoire sociale. Membre et animatrice à l'Association Canadienne pour la Santé Mentale, filiale de Montréal. Formatrice à la Table de Concertation des Organismes au Service des Personnes Réfugiées et Immigrantes (TCRI).

Orcid: 0000-00034849-6159. <dialaroche@ sympatico.ca>. que segue apresenta, no primeiro momento, a abordagem, seus fundamentos teóricos e metodológicos. No segundo momento, relata a maneira pela qual os participantes realizaram a proposta e os efeitos perceptiveis a partir de seus testemunhos. A abordagem ilustra o interesse de desenvolver uma clínica da complexidade no âmbito do domínio da saúde mental.

Palavras-chave: Sociologia clínica. Maison Saint-Dominique. Saúde mental. Clínica da complexidade. Suporte psicossocial.

\section{Le projet, la démarche la méthode}

Présentation de la MSD et du Programme "Rétablissement et Projet de Vie"

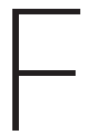
ondée en 1987, la Maison Saint-Dominique (MSD) propose des logements de longue durée, à coût abordable, ainsi qu'un soutien psychosocial à des adultes économiquement défavorisés et vivants avec des problèmes de santé mentale. Elle a pour objectif de favoriser l'autonomisation de ses résidents, les aider à développer leur plein potentiel et contribuer à leur réintégration sociale.

Elle se caractérise par un accompagnement "existentiel" pour permettre aux résidents de retrouver une vie sociale. Comme le soulignait avec force Robert Castel, I'individu a besoin de supports pour avoir une existence sociale. La MSD permet aux résidents de bénéficier de ces supports en termes de logement, d'insertion professionnelle, de vie sociale, mais aussi par un accompagnement individualisé. Le rétablissement en termes de santé mentale ne met pas au centre, comme dans les approches médicales et psychiatriques, les médicaments et la psychothérapie, mais la réinsertion sociale et la vie communautaire. Si un suivi thérapeutique est nécessaire, il n'est pas l'élément central, mais un facteur parmi d'autres. L'essentiel est de permettre aux résidents d'advenir en tant que sujet dans tous les registres de leur existence.

L'ensemble des intervenants ont manifesté un intérêt pour la sociologie clinique dans la mesure où le projet initié au départ par Marie-Maud Sylvestre-Audette (directrice) et Huguette Guay (responsable de la formation de la MSD) rejoint sur bien des points les orientations théoriques et méthodologiques développées dans des ouvrages comme L'histoire en héritage, La lutte des places, Qui est "Je"?, La part de social en nous. Tous les intervenants ont fait la même démarche préalablement aux résidents, avec Diane Laroche. Certains d'entre eux ont participé à des groupes d'implications et de recherche avec Vincent de Gaulejac et Diane Laroche. Ces expériences partagées ont conduit à l'idée du "Programme Rétablissement et Projet de Vie" qui s'est déroulé du $1^{\text {er }}$ février au 30 mars, sur huit jour- 
nées avec deux temps forts. L'un consacré au thème "Trajectoire sociale et quête de sens", à partir de la démarche "Roman familial et trajectoire sociale" initiée par Vincent de Gaulejac (1991). L'autre consacré à l'émergence d'un projet existentiel lors de deux ateliers. Le premier est la recherche d'un modèle de projet idéal déposé en eux et le second est l'élaboration d'un projet de vie personnel existentiel initié par Diane Laroche et développé à l'Université Téluq dans le cours "Sens et projet de vie".

La présentation de ce projet a été faite en interne de la MSD. Elle a suscité beaucoup d'interrogations du côté des intervenants comme des résidents. Soulignons que cette implication réciproque a été une clé de son acceptation par tous et de sa réussite. Chacun pouvant se sentir dans une situation d'égalité et de parité dans le projet. Des craintes se sont exprimées, du côté des résidents, autour de la peur d'exposer son histoire devant un groupe, l'inconfort de devoir choisir de laisser tomber une autre activité, l'appréhension de ne pas bien répondre aux attentes des intervenants, l'incertitude de ne pas se sentir assez bien pour participer à l'ensemble du projet. Du côté des intervenants, autour du sentiment que les résidents étaient trop fragiles pour un travail suivi, intense, sur plusieurs jours, nécessitant une grande concentration et une disponibilité mentale et psychique importante. Ces craintes se sont exprimées au moment du recrutement. Le projet prévoyait au départ d’impliquer huit résidents. En fait six ont décidé d'y participer au départ et cinq se sont effectivement investis. Le chiffre peut sembler faible. Mais il s'agit d'une première expérience, dont le caractère novateur et expérimental nécessitait de prendre certaines précautions et de ne jamais forcer les choses. Beaucoup doutaient, au départ, de la possibilité de le mener à bien. À plusieurs reprises les intervenants comme les résidents s'étonnaient qu'une telle démarche soit possible, et se félicitaient d'y participer, comme s'ils étaient en train de vivre un évènement exceptionnel.

\section{Présentation du}

Groupe d'Implication et de Recherche (GIR)

Le GIR est un dispositif méthodologique permettant, dans un même espace-temps, de concilier des exigences contradictoires entre recherche et implication, analyse de processus et travail sur soi, entre réflexions théoriques et prise en compte de l'expérience vécue, entre souci d’objectivation et écoute de la subjectivité.

Différents supports sont utilisés comme le prénom, le dessin du projet parental, l'arbre généalogique, la trajectoire sociale, le dessin sur le projet idéal, le dessin sur le projet personnel existentiel. Des moments de "résurgences" ponctuent chaque journée: il s'agit d'une mise en commun des résonnances diverses - physiques, 
psychiques, mentales, émotionnelles - que suscite le travail du groupe pour chaque participant. Ces moments permettent une régulation approfondie sur deux axes: le niveau d'implication individuelle au regard de l'implication collective du groupe; l'articulation entre le vécu de chacun, à partir de l'exploration de son histoire, et les réflexions théoriques sur les processus à l'œuvre.

L'objectif méthodologique consiste à créer les conditions d'un double mouvement de distanciation et d'implication à chaque étape du travail. Distanciation qui permet d'objectiver les éléments contextuels de l'histoire personnelle et d'analyser dans quelle mesure elle se trouve conditionnée par le contexte socio-historique dans lequel elle s'inscrit. Chaque destinée individuelle est le produit d'évolutions qui traversent l'ensemble des membres d'une classe sociale, d'une culture, d'une époque. II s'agit de percevoir dans quelle mesure les souffrances, les ruptures, les conflits vécus sont l'expression individualisée de contradictions sociales et de processus collectifs.

Les hypothèses produites sur les composantes socio-historiques de l'histoire individuelle ne sont validées qu'à partir du moment où elles produisent du sens pour la personne elle-même. Le cadre du séminaire est construit pour permettre une lecture plurielle de l'histoire, en jouant en permanence pour déconstruire les oppositions simples qui sous-tendent les grilles de lecture habituelles: entre la psychologie et la sociologie, l'individuel et le collectif, l'émotionnel et l'intellectuel, le social et le psychique, le verbal et le non-verbal, l'éprouvé et le réflexif. Les participants appliquent aux récits sur leur propre histoire la démarche de la recherche clinique et de l'analyse dialectique. Les supports méthodologiques proposés visent à produire du matériel sur les situations de chacun, matériel qui est analysé par l'ensemble du groupe. Chaque personne est alternativement sujet et objet de la recherche jusqu'au moment où cette opposition se transforme pour devenir simultanée. Le sujet émerge dans la capacité qu'il développe de comprendre les contradictions de son existence et de dépasser les conflits que celles-ci engendrent.

Le fait d'analyser en quoi l'individu est programmé par son histoire ne change pas cette histoire. Par contre, cela peut changer le rapport du sujet à son histoire. Dans la prise en compte des dimensions sociologiques et historiques des destins personnels - le sien, mais également ceux des autres -, chacun peut comprendre en quoi différents facteurs ont été agissants pour lui, comment ils ont contribué à le positionner dans sa famille et dans la société.

Cette démarche se situe à la frontière entre la recherche et la thérapie. La plupart des exercices proposés portent sur un travail de localisation: où suis-je dans le désir 
de mes parents? Où suis-je dans la saga familiale? Où suis-je dans les différents courants idéologiques? Où suis-je dans la structure sociale? Où suis-je dans ma généalogie? Le sujet s'aperçoit ainsi de son implication dans ces différents registres existentiels et que chacune de ces positions entretient par ailleurs des liens d'interdépendance avec les autres. En ce sens, il s'agit d'un travail sur les conflits que rencontrent les individus dans leur trajectoire et d'une analyse des contradictions qui les ont provoqués.

La dynamique entre implication et recherche construit un espace de travail intermédiaire entre deux types d'exploration. Celle qui consiste à faire un travail sur soi et qui ressemble à ce qui est fait dans un cadre thérapeutique. Mais ce travail sur soi ne consiste pas à raconter son histoire. II consiste à faire une recherche sur la façon dont le sujet, le "Je", s'est construit au carrefour des conflits et des contradictions qui ont ponctué son histoire personnelle, familiale et sociale. II s'agit donc d'un travail de recherche, d'exploration à la fois existentiel et réflexif, à partir d'une méthodologie qui est tout à la fois une méthode d'intervention - d'accompagnement du travail personnel de chacun - et une méthode de recherche construite sur des hypothèses théoriques. Le travail sur soi (implication) se définit comme une démarche clinique. Le travail de recherche s'appuie sur des hypothèses théoriques à partir de référents qui empruntent à la sociologie, mais également à d'autres disciplines - comme la psychanalyse, la psychologie, l'anthropologie, l'histoire. Dans le temps, un corpus théorique s'est constitué sous la forme d'un ensemble de théories intermédiaires sur des processus sociopsychiques que l'on observe dans ces groupes et qui reviennent de façon répétitive. L'hypothèse construite à partir d'un cas peut alors être généralisée lorsqu'on la repère de façon systématique pour tous ceux qui vivent des situations similaires.

Les groupes d'implication et de recherche sont des espaces de travail sur les conflits vécus rencontrés dans des situations sociales concrètes. Le travail en groupe conduit à une confrontation entre deux logiques cognitives. L'une s'inscrit dans une épistémologie clinique qui consiste à explorer le vécu de chaque participant à partir de supports construits pour aborder tel ou tel aspect de son histoire: le choix du prénom, l'arbre généalogique, les trajectoires professionnelles... L'autre s'inscrit dans une visée compréhensive à partir de référents théoriques issus des sciences sociales. 


\section{La méthode}

Le processus mis en œuvre se caractérise par recherche de cohérence, à toutes les étapes, entre les objectifs énoncés, le cadre mis en place et la posture des intervenants. L'essentiel est de mettre en harmonie ce qui est dit, pensé, fait et éprouvé. Les enjeux de transferts et de contre-transferts sont ici essentiels.

a. Les règles

Au démarrage du groupe d'implication et de recherche, un certain nombre de règles sont énoncées:

- Chacun est invité à travailler sur son histoire, sans forcer ni retenir ce qu'il a envie d'explorer: on s'autorise à dire, mais pour s'autoriser à dire il faut s'autoriser à ne pas dire.

- La confidentialité est essentielle pour établir la confiance: chaque participant - comme les intervenants - s'engage à ne pas répéter à l'extérieur les informations personnelles entendues dans le groupe.

- Nous sommes là non pour évaluer, mais pour comprendre. Dans les commentaires sur les histoires de chacun, on s'abstiendra de prononcer des jugements de valeur.

- L'écoute clinique consiste à se centrer sur la personne, à être attentif à ne pas la heurter. Nous ne sommes pas là pour effectuer une enquête, mais pour accompagner chacun dans l'exploration de son histoire.

- La confiance est essentielle pour que "l'enveloppe groupale" apporte la sécurité et la bienveillance nécessaire à la démarche.

- La démarche n’a pas de visée thérapeutique, mais peut avoir des effets thérapeutiques, car elle permet de fabriquer du sens.

Tout au long du processus des moments de "résurgences" sont prévus pour vérifier la cohérence du cadre et l'application de ces différents points.

\section{b. Le va-et-vient entre implication et recherche}

Chaque participant est invité à fabriquer des supports écrits (trajectoire sociale, dessin du projet parental, arbre généalogique, dessin sur le projet idéal, dessin sur le projet existentiel) à les fixer sur les murs de la salle, et à les commenter. L'im- 
plication se développe en deux temps. Dans un premier moment, chacun travaille pour lui, pour explorer son histoire, pour en repérer les éléments marquants, pour mieux saisir en quoi son histoire est toujours présente. Nous demandons à chacun d'être attentif à ses sentiments, ses émotions, ses réactions corporelles. Dans un deuxième temps, chacun expose devant le groupe son support, ce qu'il a voulu écrire et/ou dessiner, les éléments importants de son histoire.

À l'implication individuelle, répond comme écho et caisse de résonnance l'implication du groupe. D'abord dans l'attention du groupe à la présentation narrative de chacun. Ensuite dans les commentaires proposés. La posture des animateurs est elle aussi impliquée et impliquante. Dans un premier temps ils accompagnent les participants dans la fabrication des supports et l'exploration des différentes histoires. Dans les commentaires, ils favorisent l'analyse de liens, de commentaires et formulent des hypothèses qui éclairent les conflits et les contradictions vécues. Mais également sur les liens entre les contradictions du passé et les conflits du présent.

\section{c. Présentation des supports}

- Les prénoms: au démarrage, chacun est invité à se présenter en racontant ce qu'il sait de l'histoire de son prénom: "Que savez-vous des raisons pour lesquelles vos parents ont choisi votre prénom?"

$\checkmark$ La trajectoire sociale.

- Le projet parental: un dessin à partir de la consigne: "Qu'est-ce que mes parents voulaient que "Je" devienne?

- La généalogie: reconstituer sa généalogie sur trois générations. Préciser pour chacun leur statut social (catégorie socio-professionnel), les signes particuliers, les évènements marquants, les secrets.

- Le modèle de projet déposé en vous. Un dessin sur le projet idéal: dessinez vos parents qui réalisent un projet idéal, pour eux. Un projet qui leur tenait vraiment à cœur. Cela peut être aussi un projet idéal pour la mère ou un projet idéal pour le père, ou un projet qu'ils avaient en commun.

- L'anticipation et l'élaboration de votre projet existentiel: l'exercice consiste en une imagerie mentale dirigée durant laquelle les participants sont invités à explorer - "Tout au fond de moi, quel est mon désir de projet de vie?". 
- La carte d'identité idéologique: exploration des valeurs, des croyances et des engagements religieux, politiques et associatifs.

- La fiche synthèse d'accompagnement du projet existentiel: nommer le type de projet, sa direction, donner à sa vie pour une période de deux à cinq années, les objectifs, moyens et actions à prendre à court, moyen et long termes.

Ces exercices furent répartis sur huit journées, avec en préalable des rencontres individuelles et deux rencontres de groupes pour constituer le groupe final des cinq personnes impliquées dans la démarche. Deux premières journées ont été consacrées à l'exploration des prénoms et de la trajectoire sociale. Un deuxième regroupement de trois jours a permis d'explorer "le projet parental" ainsi que "I'arbre généalogique". Deux autres journées ont été consacrées au dessin sur le projet idéal, à l'élaboration du projet existentiel et à la carte d'identité idéologique; une dernière journée à la fiche synthèse d'accompagnement. Cette fiche sera utilisée mensuellement lors des rencontres de groupe en présence d'une intervenante de la Maison Saint-Dominique, choisie par les participants, qui agira comme personne ressource et accompagnera la réalisation du projet existentiel.

\section{d. Les résurgences et régulation du groupe}

À chaque étape du processus, un moment est prévu pour discuter du travail, des réactions qu'il suscite, des commentaires et des questions qu'il soulève, des sentiments qu'il génère. Le terme de résurgence évoque l'idée de faire remonter à la surface les échos, les associations, les retours réflexifs, les émotions, les résonnances psychiques, provoqués par l'exploration du roman familial et de la trajectoire sociale de chacun. Moment essentiel qui permet une régulation en continu sur deux plans. Au niveau individuel pour permettre à chacun de déposer dans le groupe ses émois, angoisses, questions, et en définitive de rendre compte du processus de désincorporation évoqué plus haut. Par ailleurs, les résurgences permettent une mise en commun de l'élaboration mentale et de la perlaboration psychique effectuée par chacun.

Ça travaille. C'est dire qu'un travail interne est à l'œuvre à tout moment et qu'il est essentiel de l'expliciter et de le partager. Les échanges dans le groupe sur la façon dont chacun est ainsi mis au travail, fait caisse de résonnance qui favorise l'harmonie groupale, l'implication personnelle et la réflexivité collective.

ces provoquent un soulagement des tensions et des doutes. Elles permettent d'expliciter la démarche, de vérifier sa cohérence et de donner des outils réflexifs sur 
ses effets. Les mots pour le dire sont essentiels: trouver les bonnes formulations sur ce que chacun ressent, sur les questions qu'il se pose, sur les émotions et les sentiments qui l'habitent.

\section{e. L'évaluation}

À la fin du groupe, un moment d'évaluation de la démarche et de ses effets est prévu. Chaque participant est invité à prendre la parole sur deux points:

- expliciter ce qu'il retient du travail fait en commun, ce qui l'a marqué et les questions qu'il se pose (versus travail sur soi);

- expliciter ce qu'il pense des supports, de la dynamique du groupe, de la posture des animateurs et de l'organisation du cadre.

Réjean, un résident, demande la parole:

Ce qui m'a plu dans cette démarche, c'est le sentiment que nous avons été toujours au centre. Quand on va voir le psychiatre, c'est lui qui est au centre. Nous n'avons jamais le temps de raconter notre histoire. II nous donne des médicaments et passe au suivant. Ici, c'est nous qui sommes au centre.

Dino prend à son tour la parole:

J'ai appris à connaitre les autres. Je me sens moins seul. On ne se parle pas pour régler des comptes.

Cette reconnaissance d'avoir été sujet dans la démarche, de pouvoir rencontrer l'altérité sans se sentir menacé sont de beaux échos du travail effectué. Chacun des participants n'est pas seulement un acteur, mais un auteur qui participe à construire le cadre et qui retrouve une puissance d'agir sur sa propre existence. II n'est plus l'objet d'un traitement, il est sujet de son histoire. II n'est plus seul, il se sent appartenir à un collectif.

\section{Témoignages et}

effets de la démarche

Dans cette deuxième partie, nous allons présenter quelques vignettes cliniques qui rendent compte des effets de la démarche pour les participants. La caractéristique de la sociologie clinique est d'éviter le double piège du vécu sans concept et du concept sans vie, selon la belle expression d'Henri Lefebvre. Dans cet esprit, 
nous alternerons l'exposé du travail effectué dans le groupe d'implication et de recherche et l'exposé de questions théoriques et/ou méthodologiques.

Le diagnostic:

un évènement biographique

La peur que suscite la démarche est bien "normale", dans la mesure où l'idée même d'un groupe d'implication et de recherche provoque, auprès de tous les publics, une ambivalence entre l'intérêt qu'il suscite et les craintes qu'il génère. L'idée même d'implication en groupe n'est pas évidente. Parler de soi n'est pas chose facile. Le faire devant un groupe encore moins. Surtout s'il s'agit de se dévoiler, de parler de ses conflits personnels, de ses failles, de ses vulnérabilités. Il convient donc de créer la confiance, de construire un cadre contenant, de ne jamais forcer les choses, de créer les conditions d'une implication réciproque et durable.

La difficulté de parler de soi sous le regard d'autrui est d'autant plus grande que le diagnostic de malade mental fait basculer l'existence dans la honte. L'intérêt de la démarche MSD est de vouloir sortir de la problématique de la maladie mentale. C'est un point capital. D’autant plus que la plupart des résidents concernés ont été, à un moment de leur parcours, sans doute désigné comme psychotique, bipolaire, schizophrène, dépressif (donc comme "fou" - explicitement ou implicitement). Cette assignation identitaire négative est lourde à porter et, pour certains, dévastatrice:

Voilà ce que je suis désormais: un malade mental.

N'oublions pas pour autant que ce diagnostic peut aussi soulager le mal-être ressenti en établissant une cause médicale qui peut donner du sens face à des tourments internes incompréhensibles.

Le récit de Julie montre que le "diagnostic" représente un tournant de la vie, un basculement, un moment clé de sa trajectoire. Mais quels que soit les circonstances, la peur de la folie et la honte d'être désigné comme tel, sont deux sentiments violents. Comment aborder cette violence sans la renforcer? II nous faut approfondir le repérage des mécanismes de défense mis en place par rapport à l'angoisse que la maladie mentale génère et réfléchir sur les processus de dégagement qui peuvent restaurer l'estime de soi.

Le travail sur la trajectoire apporte un élément essentiel. Il permet de reconsidérer le diagnostic comme un événement biographique parmi d'autres. II y a eu un avant 
et un après. Toute l'existence ne peut se réduire à cet événement, fût-il traumatique. Le partage dans le groupe de la façon dont chacun a été confronté à cette question est tout aussi déterminant. II doit favoriser un processus de dédramatisation et en relativiser les conséquences. La proposition de revenir à l'histoire familiale, provoque des réactions contrastées. Certains veulent se souvenir, d'autres préfèrent oublier.

Pierre a une belle formule pour exprimer son ambivalence:

J'ai été impassiblement secoué, car il y a des souvenirs qui ne voulaient pas revenir.

Réjean exprime bien la difficulté pour lui de notre proposition:

Ça fait des années que je veux oublier.

L'hypothèse selon laquelle l'individu est le produit d'une histoire peut provoquer des réactions opposées. Certains trouvent dans cette histoire des ressources pour mieux comprendre leurs conflits internes et se projeter dans un avenir qui ne soit plus dans la répétition du passé. D’autres y voient des empêchements, des blessures, des violences qu'ils ont besoin d'oublier, de rejeter, pour espérer se construire autrement. En définitive, l'essentiel est d'accompagner chaque participant pour lui permettre d'advenir en tant que sujet de cette histoire, dans ses aspects positifs comme dans ses aspects négatifs. L'histoire est ce qu'elle est, on ne peut rien y changer. La seule chose sur laquelle "Je" (le sujet) peut agir, c'est la façon dont cette histoire est agissante en soi.

Le travail avec Julie, la première à présenter sa trajectoire, a permis de rendre la démarche proposée effective, de lui donner une consistance, une réalité. Julie a abordé de front la question du diagnostic, de la maladie, jusqu'à évoquer une psychose majeure. Elle vérifie alors qu'elle ne met pas les autres participants en difficulté. Beau moment qui permet au groupe de se constituer et de vérifier qu'il peut être contenant. Les autres participants la rassurent en témoignant beaucoup d'intérêt et d'admiration. La confiance est au rendez-vous. Le renversement clinique aussi: l'aide et le soutien viennent des participants eux-mêmes, et du cadre qu'ils ont aidé à coconstruire. Les hiérarchies habituelles entre le savoir professionnel et le savoir existentiel, expérientiel, entre ceux qui maîtrisent le discours savant et ceux qui vivent les problèmes, entre l'élève et le "maître", commencent à s'atténuer, sinon à disparaître. 
Le récit partagé centré sur ce que chaque sujet fait de ce qu'on a fait de lui, pour reprendre la belle formule de Jean Paul Sartre, est un élément essentiel de ce processus collectif. Chaque récit apporte "des leçons" à tous. Chaque récit apporte des éléments aux autres pour affronter ses problèmes. Pierre l'évoque avec enthousiasme à propos du récit de Julie:

C'est fabuleux de voir comment elle a pu rebondir et explorer différents champs artistiques. C'est remarquable, elle a trouvé un créneau par l'art.

Bel exemple de mécanisme de dégagement face à la souffrance psychique: I'art est un moyen d'exprimer ses angoisses, ses troubles, ses problèmes, non pas dans un repli sur soi dévastateur, mais dans une création qui permet à la fois une restauration narcissique et une opportunité de resocialisation.

\section{Qu'est-ce qu'un sujet?}

Lorsque je présente l'hypothèse selon laquelle "I'individu est le produit d'une histoire dont il cherche à devenir le sujet", Diane me demande afin que je développe:

\section{C'est quoi être sujet?}

1. Simone de Beauvoir (1949) disait: "On ne naît pas femme, on le devient".

2. Cf D. Winnicott (1975). Jeu et réalité, l'espace potentiel.
Je réponds à la question en expliquant le processus qui conduit chacun de nous à réfléchir sur son histoire, sa trajectoire, ses choix, à entrer dans une réflexion sur son existence. Advenir comme sujet, c'est affirmer le "Je" comme dans la célèbre formule de René Descartes "je pense donc je suis", ou encore celle de Sigmund Freud "là où Ça était, Je dois advenir". On ne naît pas sujet, on le devient ${ }^{1}$.

Je demande au groupe si mes explications sont claires. Le groupe va alors entrer dans un jeu avec le "Je", désigné à la troisième personne du singulier. Bon, dit l'un d'entre eux,

$$
\begin{aligned}
& \text { Je propose d'aller prendre un café. } \\
& \text { Je est d'accord avec ta proposition - dit un autre. } \\
& \text { Je propose que nous fassions une pause... } \\
& \text { Je me propose d'aller aux toilettes. }
\end{aligned}
$$

Pendant quelques minutes, les "Je" vont advenir comme expression de la volonté de chacun, comme manifestation d'une volonté, d'un désir, d'une aspiration. Le jeu avec le "Je" illustre l'intérêt d'un espace transitionnel entre l'intériorité et l'extériorité, entre l'imaginaire et la réalité2. L'ouverture vers un espace de liberté et de créativité 
dans lequel chacun peut mettre en scène ses affects, ses aspirations, ses désirs, les révéler, les exprimer, les exposer aux autres, sans se sentir mis en vulnérabilité. S'affirmer comme sujet sans se sentir menacé et sans menacer l'autre. De grands éclats de rire ponctuent les formules de chacun où le "Je" se révèle. Jouer avec le "Je" donne une liberté rarement éprouvée lorsque pendant des années le "Je" n’a pas pu s'exprimer, parce qu'il a été réprimé, s'est mis en retrait ou s'est dissimulé derrière un faux self. La sensation de sortir de son Moi, pour entrer dans une expression du Je/Nous est un moment de grâce qui s'est répété à quelques reprise durant les journées suivantes de travail ensemble. La confiance s'établit, l'enveloppe groupale se consolide, la capacité de chacun à s'exprimer et prendre la parole se développe.

\section{La dynamique entre travail sur soi (pour soi) et réflexion collective}

On travaille fort ici parce qu'on s'en parle tous ensemble - dit l'une des participantes -, et tout le monde a accepté tout le monde.

La dynamique du groupe est une aide lorsqu'elle vient soutenir les dynamiques individuelles de chacun, la possibilité de s'exprimer, les interrelations, mais aussi les rapports avec les animateurs. Le groupe est une instance qui favorise les processus de triangulation, donc de symbolisation. Chaque participant est invité à sortir de son théâtre intérieur, du face à face avec lui-même. La construction des différents supports facilite la mise en forme du récit de son histoire personnelle et familiale. Il le met en ordre pour lui-même avant de pouvoir l'exposer à d'autres? Cette mise en forme et en mots, favorise les capacités narratives dans un double mouvement d'élaboration mentale et de perlaboration psychique ${ }^{3}$. Entre imaginaire et réalité, la symbolisation permet de développer un espace entre le moi et le monde nécessaire pour amortir les angoisses, les affects, les débordements émotionnels.

Replonger dans son histoire n'est pas sans risque. Le sujet peut le vivre comme une régression menaçante dans la mesure où il va retrouver les traumatismes, les conflits, les violences vécues dans l'enfance. Comment faire pour se dégager de cette violence vécue dans le passé? Comment faire pour s'y confronter sans retomber dans la souffrance qu'elle a pu provoquer au moment où le sujet l'a rencontré? Comment travailler sur la violence sans violence? Si l'histoire est "incorporée", il convient de mettre en place un cadre favorisant la désincorporation. Les supports, l'exploration collective, le transfert sur des animateurs institués comme figures protectrices, l'empathie et la bienveillance qui constituent une enveloppe groupale contenante, la mise en mot de son histoire, la formulation d'hypothèses sur les liens entre les contradictions rencontrées dans le passé et les conflits vécus dans le

3. La perlaboration
désigne le travail
psychique effectué
en psychanalyse
qui permet au
patient d'intégrer
une interprétation
et de surmonter
les résistances
qu'elle suscite. En
anglais le terme
working-through
rend mieux compte
de l'idée qu'il s'agit
d'un travail du sujet
qui affronte ses
difficultés internes,
ses défenses
inconscientes, pour
reconnaitre ce qu'il
vit à l'intérieur de
lui, recomposer les
éléments de son
histoire, et accepter
de l'exposer à
d'autres.

3. La perlaboration désigne le travail psychique effectué en psychanalyse qui permet au une interprétation et de surmonter anglais le terme working-through rend mieux compte d'un travail du sujet qui affronte ses difficultés internes, ses défenses inconscientes, pour reconnaitre ce qu'il I'intérieur de éléments de son histoire, et accepte d'autres. 
présent sont autant d'éléments qui permettent à chacun de "sortir de lui-même" par un processus de symbolisation active.

On se surpasse ici - dit Suzanne - à la fin de la première journée, on est capable d'aller au-delà de ce qu'on est.

Cette idée de surpassement illustre la force que suscite le sentiment d'advenir comme sujet, de reprendre sa vie en main, de retrouver une puissance d'agir sur son existence. Au Moi balloté par une histoire subie, le sujet révèle une capacité "d’aller au-delà" de ce qu'il est.

\section{L'expression verbale et non verbale}

Lorsque nous donnons la consigne "dessiner ce que mes parents voulaient que Je devienne", Suzanne nous dit:

Je ne sais pas dessiner.

Cette réaction est révélatrice du refoulement de la fonction imaginaire. Le sujet ne se sent pas en capacité de mettre des images sur son histoire, sa vie, ses aspirations. II n'arrive pas à s'imaginer autre chose que ce qu'il est. Ce refoulement bloque les capacités de remémoration comme celle de pouvoir se projeter dans un avenir désirable. Il empêche le sujet de rêver sa vie, de s'imaginer une autre vie, de se projeter dans un projet de vie souhaitable, qui vaut la peine d'être vécue. Le dessin favorise la projection de soi-même dans un imaginaire moteur - selon l'expression d'Eugène Enriquez - afin d'échapper à un imaginaire bloqué qui réduit le possible au probable.

Dans un deuxième temps, Suzanne accepte d'entrer dans l'exercice proposé. Elle écrit:

Ma mère voulait que je devienne hôtesse de l'air.

Puis elle "ose" dessiner un avion, puis mettre des couleurs, un avion avec des fenêtres. Peu à peu, l'expression se libère, et Suzanne exprime sa joie de dessiner, de colorier. Elle retrouve le plaisir d'une expression moins retenue, d'un plaisir moins contenu, de faire de sa vie un dessin, un dessein. Dans les commentaires, on entendra même l'idée qu'avec son avion, Suzanne s'autorise "à s'envoyer en l'air". Expression, sans doute un peu cavalière, qui révèle combien c'est le désir du sujet qui peut s'exprimer par cette levée du refoulement de l'imaginaire. Suzanne viendra confirmer ce sentiment de liberté retrouvée en évoquant ses aspirations refoulées:

Je voulais être mannequin et chanteuse, comme dans les revues. J'ai l'entregent pour faire ça. 
Et elle évoque son activité de vendeuse de revues, l'itinéraire, qu'elle distribue avec plaisir parce qu'elle fait rire les clients potentiels. Elle est fière de cette activité qui lui donne un statut respectable et lui procure des revenus.

Nous avons été frappés par le soin avec lequel les participants s'exprimaient, l'attention à bien choisir les mots pour présenter leur récit, réagir aux présentations des autres. Ils ne parlent jamais pour ne rien dire. Ils ont besoin d'une cohérence entre le mental (élaboration réflexive) et le psychique, le ressenti, l'éprouvé. Dans un premier temps, c'est l'écoute qui prime. Écoute des objectifs, des consignes, des règles de fonctionnements. Ils sont concentrés, attentifs, habités par le souci de bien faire. Le respect mutuel, l'attention aux autres, la réciprocité des implications. Ils se sentent soutenus et contenus par le groupe. Jamais un mot d'évaluation déplacé. Dans un premier temps nous pouvons penser qu'il s'agit de contenir l'anxiété face à l'inconnu. Le "recrutement" des participants a montré que l'engagement dans le projet a suscité, au sein de la MSD, beaucoup d'interrogations et d'anxiété. On peut comprendre que l'implication demandée, le fait d'avoir à parler de soi devant un groupe, de raconter son histoire familiale suscitent bien des réserves sinon des craintes. Celles-ci se sont atténuées au fur et à mesure du processus jusqu'à l'évaluation finale qui a été très positive. "C'est un défi qu'il nous fallait relever" dira l'un d'eux, "une épreuve", dira un autre, qui en définitive aura été bénéfique.

\section{L'incorporation de l'histoire}

Le cadre et les supports construisent un espace d'exploration entre le réel, l'imaginaire et le symbolique. Ils favorisent un va-et-vient continu entre la scène intérieure et la scène sociale entre le fantasme et la réalité, entre les affects éprouvés et la mentalisation.

Avec des amis, on ne parle pas comme ça - dira Julie. Ici, on prend le temps, on comprend les choses de façon moins émotive.

Et Pierre de poursuivre:

Les exercices m'ont replongé dans des souvenirs douloureux, mais il y a une grosse libération d'y être retourné, même si sur le coup, ça fait mal.

Pour la plupart des résidents, la maladie mentale opère une fixation dans le présent qui fige la capacité à se projeter dans l'avenir, comme la possibilité de se remémorer le passé, vécu avant tout dans le registre traumatique. À quoi peut bien servir le fait de se replonger dans une histoire lourde de souvenirs douloureux, de sépa- 
4. L'expression est de Max Pagès (1993).

rations déchirantes, de crises récurrentes, des passages à l'acte destructeur? On ne peut modifier le passé. Par contre, on peut changer son rapport au passé, à la façon dont l'histoire est incorporée en soi, atténuer ses effets destructeurs.

L'histoire est inscrite dans le fonctionnement psychique et dans la mémoire. Les évènements biographiques traumatisants provoquent des effets de sidération qui se fixent dans l'inconscient. Ils s'installent sous forme de connexions gelées ${ }^{4}$ qui figent la vitalité du sujet.

Le terme "gelé", appliqué au fonctionnement psychique est inhabituel, mais intéressant. Quand le sang se remet à circuler dans un membre gelé, la douleur est intense alors même que les gelures le rendaient insensible. Mais le fait que le sang se remette à circuler est une bonne chose, c'est la santé qui revient. II en va de même pour les éléments traumatiques de l'histoire qui s'enkystent dans le psychisme. Comme le dit Pierre, la reviviscence de souvenirs douloureux fait mal, mais la libération qu'elle engendre fait du bien. La douleur est un symptôme, une alerte que quelque chose ne va pas. Ce n'est donc pas elle qu'il faut supprimer, elle n'est qu'un effet, mais les causes qui la provoquent.

\section{L'inversion de la honte en fierté}

Réjean n'a pas connu son père. Mais sa mère voulait qu'il ait une activité manuelle. Lui revient alors à la mémoire que son père était charpentier. II fait alors le lien entre le projet maternel et l'activité paternelle.

\footnotetext{
Je n’avais pas réalisé ça-nous dit-il. À cinq, six ans je voulais devenir avocat pour défendre mes frères qui avaient des bisbilles, sans savoir qu'il fallait aller à l'université. Plus tard, j'ai défendu un de mes frères qui a fait quelque chose de grave. J'ai eu beaucoup de mal dans ma vie, j'essaie aujourd'hui de faire quelque chose de bien. Je n'ai plus le cerveau pour faire des études. Mais à la Maison Saint Dominique, ils m’ont sauvé la vie, ils m'ont donné beaucoup de chances. Un de mes projets de vie, c'est d'aider les autres à gagner leur vie.
}

Si l'expression signifie dans le sens courant trouver du travail, on peut l'entendre ici comme un moyen de sortir d'une vie de perdant (looser) pour commencer une vie de gagnant (winner). Le témoignage de Réjean est salué par les autres membres du groupe comme une rédemption. Chacun reconnaît sa disponibilité et sa générosité au sein de la MSD. Après une vie chaotique, marqué par la violence, la délinquance, la prison et de nombreux épisodes psychiatriques, il se fait du bien en faisant du bien aux autres. Cette inversion transforme la culpabilité en altérité, la honte en 
fierté, la haine de soi en amour propre, l'assignation à une identité négative en estime de soi et des autres.

Par quelle alchimie une telle transformation est-elle possible? La MSD offre un ensemble de possibilités matérielles, et un accompagnement relationnel qui sont autant de supports affectifs. Mais cette "chance" n'est opérante que si le sujet s'en saisit. Faire confiance au sujet, en ses capacités d'advenir, de convertir des pulsions destructrices en investissement socialement utile.

\section{Causalité psychique, causalité sociale}

Réjean semble très ému lorsqu'il présente sa trajectoire. II est le dernier d'une famille de 12 enfants. II a quatre, cinq ans lorsque ses parents se séparent. II est alors placé en famille d'accueil, la première année avec ses frères et sœurs les plus jeunes, puis sans eux. À 11 ans, il revient quelque temps chez sa mère avec un de ses frères.

Ce n'étaient pas des bons souvenirs, on se disputait tout le temps.

Les services sociaux ont décidé de nous placer à nouveau. II sera ainsi ballotté de familles d'accueil en institutions, coupé du reste de sa fratrie.

Je les ai retrouvés à 14, 15 ans, parce qu'on a tous été élevés dans des familles d'accueil. C'est ma sœur Florence qui nous a tous retrouvés.

L'histoire de Réjean est marquée par l'instabilité, la violence, des séparations continuelles. II ne se plaint pas des familles qui l'ont accueilli. Mais il ne restait jamais assez longtemps pour s'attacher.

J'étais turbulent à l'école. J'étais mal accepté en classe.

On comprend là comment les conditions concrètes d'existence produisent des comportements qui vont être interprétés comme la cause des problèmes psychologiques de Réjean. Toute sa petite enfance est conditionnée par des turbulences affectives, sociales, scolaires, géographique. II n'est pas étonnant qu'il devienne un enfant "turbulent". Comme tous les enfants maltraités par la vie, continuellement changé d'environnement, d'école, de lieu, de familles, il est confronté à des problèmes d'attachement. II devient un enfant difficile, perturbé, violent, un enfant qui se fait rejeter de partout. C'est alors son comportement qui devient l'élément explicatif de sa trajectoire d'enfant placé, des turbulences dont il est l'objet. La causalité sociale de départ se transforme en causalité psychologique. Dans ces conditions, l'enfant va intérioriser le fait que c'est bien lui qui est responsable de ce qui lui arrive. II est 
mis en instabilité permanente, et on le considère comme un enfant instable. Son comportement agité, violent, insupportable va devenir le problème majeur. II va être considéré comme un "inadapté", alors que ce comportement est une adaptation à des conditions de vie agitées, violentes, insupportables. II devient un enfant à problème alors que le problème ne vient pas de lui, mais de son histoire familiale.

On peut penser que les problèmes de violence et de délinquance qui parqueront sa destinée future sont largement déterminés par son histoire chaotique. Ce qui, au départ, est la conséquence de ses conditions concrètes d'existence, donc des problèmes objectifs, vont devenir des problèmes de comportement psychologiques, donc subjectifs.

Les causalités s'entremêlent parce qu'il y a des influences réciproques entre le psychique et le social. Les processus sociaux et psychiques interfèrent en permanence pour devenir des processus sociopsychiques.

\section{Conclusion:}

pour une clinique de la complexité, une approche alternative dans un contexte de rétablissement en santé mentale

Il convient donc d'entrer dans le pluralisme causal et de sortir des oppositions habituelles entre les approches médicales, psychologiques et sociologiques. La personne humaine est une totalité bio-psycho-sociale. C'est dire que ses comportements, ses symptômes et ses conduites sont déterminés par une multiplicité de causes sans que l'on puisse dégager une instance ultime qui serait la clé explicative de l'ensemble (l'inconscient, le cerveau, les gènes, le caractère, les relations familiales, la généalogie, le milieu socio-culturel...). Chacun de ces déterminants est à la fois conditionné et conditionnant, produit et producteur dans des relations qui sont tout à la fois systémiques et récursives. Les comportements et les conduites sont toujours la conséquence d'éléments hétérogènes - génétiques, physiologiques, psychologiques, sociologiques - qui ressortent de disciplines diverses.

L'analyse de la réciprocité des influences entre les registres corporels, psychiques et sociaux est l'objet même d'une clinique de la complexité. Dans certains cas, ces influences se combinent, et se renforcent, parfois elles se neutralisent ou s'inhibent. Il ne s'agit donc pas simplement de juxtaposer les différentes approches, mais d'approfondir ce que chacune peut apporter et la façon dont on peut construire des articulations entre elles. L'idée de la "problématisation" multiple propose une démarche multipolaire qui consiste à croiser plusieurs points de vue théoriques issus de 
disciplines différentes, à adopter plusieurs perspectives, à éclairer les phénomènes étudiés à partir de problématiques issues de théories différentes, voire divergentes.

La construction d'une clinique de la complexité favorise des réflexions transversales, bouscule les frontières disciplinaires, développe les liens entre les chercheurs et les praticiens. Le projet de la MSD participe à ce projet en réaction contre les replis identitaires qui animent trop souvent les différentes approches en santé mentale. La question primordiale est de lier en permanence la recherche sur la pratique et la pratique de la recherche. La clinique de la complexité se fonde sur l'expérience pratique (la confrontation quotidienne avec le vécu des résidents et/ou des personnes que l'on accompagne), et sur la réflexion théorique (l'approfondissement des hypothèses qui fondent cette pratique). Pour développer cette orientation, il convient, de sortir de cadres trop souvent individualisant, d'ouvrir le champ de la pratique à d'autres façons de faire et de penser, d'accepter que les personnes usagères des services en santé mentale participent à définir leurs parcours thérapeutiques, donc de leur offrir des options, des combinaisons, des dispositifs pluriels.

Nous sommes bien conscients de la nécessité de protéger le cadre thérapeutique. La thérapie est un lieu qui doit être protecteur, contenant, sécurisant, construit pour accompagner des personnes en état de vulnérabilité. La thérapie est un refuge qui accueille des personnes en difficulté. Le terme de refuge est cependant ambivalent. Il exprime l'idée d'un recours, d'un lieu dans lequel on peut échapper à la violence et à la répression. Mais il connote également l'idée d'un lieu à part, isolé du monde, à l'écart. C'est une des raisons pour laquelle le social en est trop souvent exclu. La logique des approches psychiatriques, psychanalytique et psychothérapeutiques induit à un travail individualisé qui contribue à désocialiser la personne.

Si donc on peut comprendre et accepter la nécessité pour certains de se mettre de côté, de se mettre en retrait, la question se pose des conséquences de cette posture dans la pratique. Cela conduit à privilégier l'exploration des facteurs psychiques à l'exclusion des facteurs sociaux et à minimiser les questions relatives à l'insertion sociale des personnes usagères des services en santé mentale à leur réadaptation à la réalité. Pour éviter les travers liés au psychologisme, il convient donc de répondre à deux exigences contradictoires. Une exigence clinique de protection permettant au sujet un travail approfondi sur lui-même, une exigence d'adaptation au monde permettant au sujet de comprendre en quoi le social est agissant en lui.

En définitive, c'est le processus même de construction d'une clinique de la complexité qui est porteur de changement à partir du moment où il favorise l'émer- 
gence du sujet. Des sujets qui adviennent en s'impliquant dans la construction d'une clinique innovante, ouverte, qui réponde aux contradictions de notre temps.

\title{
Épilogue
}

La restitution.

\begin{abstract}
Dans la discussion, ils ont souhaité que le mot folie soit annulé et remplacé par celui de maladie mentale. Les termes de fou et de folie sont socialement stigmatisants et psychologiquement invalidants. Ils assignent le sujet dans une identité négative et menaçante. Ils mettent la personne au ban de la société dans une exclusion quasi irréversible. Ils annulent la possibilité d'advenir en tant que sujet comme s'il était dépossédé de lui-même. Le terme de maladie mentale est plus acceptable. II indique que la personne ne va pas bien, qu'il y a quelque chose en elle qui peut être soignée, qu'il s'agit d'un symptôme qui peut disparaître, évoluer, devenir l'objet d'un traitement. Le sujet n'est pas annulé, bien au contraire. Il est sollicité pour prendre en charge son traitement, se donner les moyens de se soigner, affronter le symptôme pour limiter ses effets destructeurs pour lui et pour la société.
\end{abstract}

\section{Références}

BEAUVOIR, S. de. Le deuxième sexe. Paris: Gallimard, 1949.

GAULEJAC, V. de. Qui est "Je"? Paris: Les Éditions du Seuil, 2009.

. L'histoire en héritage, roman familial et trajectoire sociale. Paris: Éditions Desclée de Brouwer, 1999.

. La névrose de classe. Trajectoire sociale et conflits d'identité suivi d'une lettre d'Annie Ernaux. Paris: Payot, 1991 [1987].

GAULEJAC, V. de; COQUELLE, C. (Orgs). La part de social en nous. Sociologie clinique et psychothérapies. Paris: Éditions Érès, 2017.

GAULEJAC, V. de; TABOADA LEONETTI, I. La lutte des places. Paris: Éditions Desclée de Brouwer, 1999.

PAGÈS, M. Psychothérapie et complexité. Paris: Éditions Desclée de Brouwer, 1993.

WINNICOTT, D. Jeu et réalité: l'espace potentiel. Paris, Gallimard, 1975. 